\title{
Alterations in Gut Vitamin and Amino Acid Metabolism are Associated with Symptoms and Neurodevelopment in Children with Autism Spectrum Disorder
}

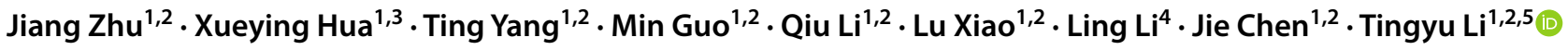

Accepted: 2 May 2021 / Published online: 14 July 2021

(c) The Author(s) 2021

\begin{abstract}
Metabolic disturbance may be implicated in the pathogenesis of autism. This study aimed to investigate the gut metabolomic profiles of autistic children and to analyze potential interaction between gut metabolites with autistic symptoms and neurodevelopment levels. We involved 120 autistic and 60 neurotypical children. Autistic symptoms and neurodevelopment levels were assessed. Fecal samples were analyzed using untargeted liquid chromatography-tandem mass spectrometry methods. Our results showed the metabolic disturbances of autistic children involved in multiple vitamin and amino acid metabolism pathways, with the strongest enrichment identified for tryptophan metabolism, retinol metabolism, cysteine-methionine metabolism, and vitamin digestion and absorption. Differential gut metabolites were correlated to autistic symptoms and neurodevelopment levels. Our findings improved the understanding of the perturbations of metabolome networks in autism.
\end{abstract}

Keywords Autism $\cdot$ Metabolomics $\cdot$ Metabolism $\cdot$ Vitamin $\cdot$ Symptoms $\cdot$ Children

\section{Abbreviations \\ 5-HIAA 5-Hydroxyindoleacetic acid \\ 5-HT 5-Hydroxytryptamine \\ 5-HTP 5-Hydroxytryptophan \\ 5-MTHF 5-Methyltetrahydrofolate}

\section{Jie Chen}

jchen010@hospital.cqmu.edu.cn

$\triangle$ Tingyu Li

tyli@vip.sina.com

1 Children's Nutrition Research Center, Children's Hospital of Chongqing Medical University, Chongqing Key Laboratory of Childhood Nutrition and Health, Chongqing 400014, China

2 Ministry of Education Key Laboratory of Child Development and Disorders, China International Science and Technology Cooperation Base of Child Development and Critical Disorders, National Clinical Research Center for Child Health and Disorders, Chongqing 400014, China

3 Department of Neonatology, Chongqing Medical University Affiliated Children Hospital, Chongqing 400014, China

4 Department of Children Rehabilitation, Hainan Women and Children's Medical Center, Hainan 570100, China

5 Director of Key Lab of Ministry of Education, Children's Nutrition Research Center, Children's Hospital of Chongqing Medical University, No.136 Zhongshan Er Road, Yuzhong District, Chongqing 400014, China
8-OHdG 8-Hydroxy-deoxyguanosine

ABC Autism Behavior Checklist

ASD Autism spectrum disorder

BMI Body mass index

CARS Childhood Autism Rating Scale

DHF Dihydrofolate

DQ Developmental quotient

GABA $\gamma$-Aminobutyric acid

GDS Gesell Developmental Scale

GI Gastrointestinal

Hcy Homocysteine

LC-MS Liquid chromatography-mass spectrometry

NAC N-acetylcysteine

PLS-DA Partial least squares discriminant analysis

SRS Social Responsiveness Scale

TD Typically developing

TPP Thiamine-pyrophosphate

VAD Vitamin A deficiency

VIP Variable important in projection

\section{Introduction}

Autism spectrum disorder (ASD) is a complex neurodevelopmental disorder characterized by early-appearing social communication deficits and restricted or repetitive behaviors 
(Hyman et al., 2020). Besides the core deficits, ASD is often accompanied with other developmental or behavioral disorders, sleep problems, and gastrointestinal (GI) symptoms (Hyman et al., 2020). Currently, the precise etiology and mechanism of ASD remain unclear, thus hindering the development of available laboratory diagnostic and effective cure for the condition (Muhle et al., 2018).

Accumulated evidence has supported metabolic disturbance may be implicated in the pathogenesis of ASD. Metabolomics studies of urines, plasma, and fecal samples from ASD patients have shown disturbances of metabolism related to amino acids, oxidative stress, purine intermediates, and gut microbiota (Glinton \& Elsea, 2019; Shen et al., 2020; Mohamadkhani, 2018; Kang et al., 2018). Despite the high interest, previous studies are mainly focused on the metabolomic analysis of urines and blood, and analysis of gut microbiota composition, while studies of fecal metabolism are relatively rare in the context of ASD (Kang et al., 2018; Shen et al., 2020).

Gut metabolomics can provide comprehensive information about the final products of interactions among dietary intake, metabolism, and microbial functions. Studies have shown that short-chain fatty acids (SCFAs) altered in the gut of ASD children (Liu et al., 2019; Thomas et al., 2012), and SCFAs could regulate gut immunity and genes expression of the host (Chang et al., 2014). Abnormal glutamate and $\gamma$-aminobutyric acid (GABA) metabolism were observed in the feces of children with ASD, which may influence excitation-inhibition balance (Kang et al., 2018; Wang et al., 2019). Abnormal tryptophan metabolism and increased serotonin (5-hydroxytryptamine, 5-HT) have been observed in in the gut of ASD patients (Muller et al., 2016). Also, isopropanol and phenol substances, including phenol and $p$-cresol, were found higher in fecal of children with ASD (De Angelis et al., 2013; Kang et al., 2018).

Although the above reports suggest that altered gut metabolomics may contribute to the pathogenesis of ASD, the specific alterations in individual compounds were inconsistent between studies owing to multiple potential confounders (e.g., ethnicity, age, diet, disease, medicine, and methodology used) that can influence the metabolism outcomes. Inconsistent and scattered changes in single metabolites have a limited role in elucidating the pathophysiology of ASD. Therefore, a comprehensive interpretation of the metabolism pathway network is required.

This study aimed to determine the gut metabolomic profiles of children with ASD and identify the potential associations of gut metabolites with ASD symptoms and neurodevelopment levels. We analyzed the fecal metabolomic profiles of preschool children with ASD and age-, sex-, region- matched typically developing (TD) children with liquid chromatography-tandem mass spectrometry (LC-MS/ MS) methods. We found that the differential metabolites between the ASD and TD groups were mainly involved in multiple vitamin and amino acid metabolism pathways. We also investigated the possible correlations of the altered gut metabolites with symptoms and neurodevelopment levels of ASD children, and postulated the interconnection of vitamins and amino acids in the metabolism network of ASD.

\section{Subjects and Methods}

\section{Subject Selection}

A total of 120 children with ASD (ages, 2-6 years) were enrolled for this study from the Maternal and Child Care Health Hospital of Hainan Province, China, after a comprehensive assessment. The inclusion criteria were a diagnosis of ASD made by a developmental pediatrician through a series of structured interviews according to the Diagnostic and Statistical Manual of Mental Disorders (Fifth Edition, DSM-5) criteria (American Psychiatric Association, 2013). The Childhood Autism Rating Scale (CARS) (Rellini et al., 2004) was used to assist diagnosis (scores $>30$ ). The exclusion criteria included other developmental disorders, neurological or psychiatric diseases, genetic metabolic disease, major physical illness, a recent history of (i.e., within 3 months before sampling) infection, special diets, or antibiotic/probiotic use.

ASD symptoms were assessed using the Autism Behavior Checklist (ABC) (Rellini et al., 2004), Social Responsiveness Scale (SRS) (Cen et al., 2017) and CARS, and higher scores indicated more severe symptoms. Neurodevelopment level in ASD children was assessed using the revised Gesell Developmental Scale (GDS) (Jin et al., 2007), which is extensively used in China to evaluate cognitive and behavioral development. Development quotient scores (DQ) of GDS were used to assess the levels of intellectual and behavioral development. DQ scores $<75$ indicated developmental delay, and the lower the DQ score, the more severe the developmental delay. Gastrointestinal (GI) symptoms were evaluated with the six-item Gastrointestinal Severity Index (6-GSI), and higher scores indicated more severe GI symptoms.

A control group of $60 \mathrm{TD}$ children was recruited and matched to the ASD group by age, gender, and region. The TD children underwent health examinations at the Department of Child Health in the Maternal and Child Care Health Hospital of Hainan Province. All control subjects were healthy, and showed no sign of developmental disorders, psychiatric diseases, or GI symptoms. Other exclusion criteria were the same as those for the ASD group.

Participation in this research was voluntary. The study protocol was approved by the Medical Ethic Committee. 
This cross-sectional study was based on a clinical trial which was registered in the Chinese Clinical Trial Registry (ChiCTR-ROC-14005442).

\section{Fecal Sample Collection and LC-MS Metabolomics Analysis}

\section{Fecal Sample Collection and Metabolites Extraction}

Fresh fecal samples from each participant were placed in sterile tubes, immediately frozen, and stored at $-80^{\circ} \mathrm{C}$ until metabolomics analysis. The $100 \mathrm{mg}$ of stool for each sample was separately ground with liquid nitrogen, and the resulting homogenate was resuspended in prechilled $80 \%$ methanol and $0.1 \%$ formic acid by vortexing thoroughly. The samples were incubated on ice for $5 \mathrm{~min}$ and then centrifuged at $15,000 \mathrm{rpm} / \mathrm{min}$ at $4{ }^{\circ} \mathrm{C}$ for $5 \mathrm{~min}$. The supernatant was diluted with LC-MS-grade water (final concentration, $60 \%$ methanol), transferred into a fresh Eppendorf tube through a $0.22-\mu \mathrm{m}$ filter, and centrifuged again at $15,000 \mathrm{rpm} / \mathrm{min}$ at $4{ }^{\circ} \mathrm{C}$ for $10 \mathrm{~min}$. Finally, the filtrate was injected into the LC-MS/MS system for analysis.

\section{LC-MS/MS Analysis}

LC-MS/MS analyses were performed using a Vanquish UHPLC system (Thermo Fisher, USA) and an Orbitrap Q Exactive HF-X mass spectrometer (Thermo Fisher). Briefly, the metabolites were separated and characterized using LC system and further detected with MS system. Samples were injected onto an Hyperil Gold column $(100 \times 2.1 \mathrm{~mm}$, $1.9 \mu \mathrm{m}$ ) at a flow rate of $0.2 \mathrm{~mL} / \mathrm{min}$ and separated using a 16-min linear gradient. The eluents for the positive polarity mode were $0.1 \%$ formic acid in water (eluent $\mathrm{A}$ ) and methanol (eluent B). The eluents for the negative polarity mode were $5 \mathrm{mM}$ ammonium acetate, $\mathrm{pH} 9.0$ (eluent $\mathrm{A}$ ) and methanol (eluent B). The solvent gradient was set as follows: $2 \%$ B, $1.5 \mathrm{~min} ; 2-100 \%$ B, $12.0 \mathrm{~min} ; 100 \%$ B, $14.0 \mathrm{~min}$; $100-2 \% \mathrm{~B}, 14.1 \mathrm{~min}$; and 2\% B, 16 min. The Q Exactive HF-X mass spectrometer was operated in the positive/negative polarity mode with $3.2 \mathrm{kV}$ spray voltage, 35 arb sheath gas-flow rate, 10 arb aux gas-flow rate, and $320^{\circ} \mathrm{C}$ capillary temperature.

\section{Metabolite Analysis}

Compound Discoverer v3.0 (CD 3.0, Thermo Fisher) was used to process and normalize the raw data files generated by UHPLC-MS/MS and to perform peak alignment, peak selection, and quantification for each metabolite. The main parameters were set as follows: retention time tolerance, $0.2 \mathrm{~min}$; actual mass tolerance, $5 \mathrm{ppm}$; signal intensity tolerance, $30 \%$; signal/noise ratio, 3; and minimum intensity,
100,000 . Peak intensities were normalized against the total spectral intensity, and normalized data were used to predict the molecular formula based on additive ions, molecular ion peaks, and fragment ions. Peaks were matched with the mzCloud (https://www.mzcloud.org/) and ChemSpider (http://www.chemspider.com/) databases to obtain accurate qualitative and relative quantitative results.

The normalized metabolism data were analyzed using the CentOS (release 6.6), R ( $v \mathrm{R}-3.4 .3)$, and SPSS ( $v 19.0$, USA). With individual metabolites dataset, Partial least squares discriminant analysis (PLS-DA) models were built to visualize the metabolic alteration patterns between the ASD and TD groups. Furthermore, the cross-validation analysis of variance was performed to assess the reliability of the models. Differential metabolites between the two groups were selected using combined multivariate and univariate analyses. Gut metabolites with a fold change $>1.5$, a variable importance in projection score (VIP score) $>1$, and a false discovery rate-corrected $p$ value $<0.05$ for the Student's $t$ test or Mann-Whitney $U$ test were considered significantly differentially expressed between the two groups. To further demonstrate the biological functions of the differential metabolites, the Kyoto Encyclopedia of Genes and Genomes (KEGG) pathways enrichment analysis was performed (http://www.genome.jp/kegg/). A hypergeometric test was used to assess the significance of the enriched KEGG pathway.

Metabolomics analysis was carried out according to the standard protocols recommended by Novogene Technology Co., Ltd. (Beijing, China).

\section{Statistical Analysis}

Demographics and clinical assessment data were analyzed using SPSS (v19.0). Continuous variables were described as the means with standard deviations or medians (interquartile ranges) as appropriate, and categorical variables were described as percentages. The two-tailed Student's $t$ test, Mann-Whitney $U$ test, and the chi-square test were used to compare between groups. Correlations of metabolites levels with clinical assessment scores were analyzed by Spearman correlation. $p$ value $<0.05$ indicated statistical significance.

\section{Results}

\section{Subject Characteristics}

A total of 120 ASD children (ages, 2-6 years) and 60 TD children were enrolled for this study. The demographic and clinical features of the participants are presented in Table 1. There were no significant differences in age-gender 
Table 1 Demographic and clinical characteristics of participants

\begin{tabular}{|c|c|c|c|}
\hline & TD & ASD & $p$ value \\
\hline Age (years), mean $\pm S D$ & $4.01 \pm 1.12$ & $3.86 \pm 1.03$ & 0.2182 \\
\hline Sex (male/female) & $39 / 21$ & $99 / 21$ & 0.079 \\
\hline \multicolumn{4}{|c|}{ Family annual income per capita (RMB), n (\%) } \\
\hline$\leq 20,000$ & $31(51.67)$ & $67(55.83)$ & 0.597 \\
\hline$>20,000$ & $29(48.33)$ & $53(44.17)$ & \\
\hline Height (ZHA) & $0.01 \pm 0.94$ & $-0.14 \pm 1.0$ & 0.2429 \\
\hline Weight (ZWA) & $-0.02 \pm 0.98$ & $0.06 \pm 0.96$ & 0.5771 \\
\hline BMI (ZBMI) & $0.26 \pm 0.92$ & $0.33 \pm 1.07$ & 0.6646 \\
\hline Picky eating, $\mathrm{n}(\%)$ & $26(43.33)$ & $80(66.67)$ & $0.003^{* *}$ \\
\hline GI symptoms, n (\%) & 0 & $58(48.33)$ & \\
\hline \multicolumn{4}{|l|}{$\mathrm{ABC}$} \\
\hline Sensory & - & $8.37 \pm 5.04$ & \\
\hline Social withdrawal & - & $14.13 \pm 7.74$ & \\
\hline Stereotypic behavior & - & $7.63 \pm 7.01$ & \\
\hline Inappropriate speech & - & $13.57 \pm 5.98$ & \\
\hline Laggard daily living ability & - & $11.28 \pm 5.18$ & \\
\hline Total ABC scores & - & $54.98 \pm 22.74$ & \\
\hline \multicolumn{4}{|l|}{ SRS } \\
\hline Social awareness & - & $11.83 \pm 3.09$ & \\
\hline Social cognition & - & $18.68 \pm 4.52$ & \\
\hline Social communication & - & $33.29 \pm 8.82$ & \\
\hline Social motivation & - & $14.96 \pm 4.4$ & \\
\hline Autistic mannerisms & - & $13.71 \pm 5.58$ & \\
\hline Total SRS scores & - & $92.47 \pm 21.65$ & \\
\hline CARS & - & $37.18 \pm 5.86$ & \\
\hline \multicolumn{4}{|l|}{ GDS } \\
\hline Adaptive behavior & - & $57.01 \pm 17.94$ & \\
\hline Gross motor & - & $64 \pm 14.22$ & \\
\hline Fine motor & - & $57.45 \pm 17.05$ & \\
\hline Language & - & $43.62 \pm 19.97$ & \\
\hline Personal-social behavior & - & $48.47 \pm 15.24$ & \\
\hline
\end{tabular}

The two-tailed Student's $t$ test, and the chi-square test were used for analysis

$T D$ typically developing, $A S D$ autism spectrum disorders. ** $p<0.01$

composition and z-score of the body mass index (BMI) between the two groups. Of the 120 children with ASD, 80 (66.67\%) showed food selectivity, and 58 (48.33\%) had GI symptoms.

\section{Alterations in Gut Metabolism Profiles of ASD Children}

To explore the gut metabolic patterns associated with ASD status, a fecal metabolome analysis was performed by LC-MS/MS method. A total of 6936 peaks of compounds were obtained, among which 4531 were explored in the positive ion mode (ESI+) and 2405 in the negative ion mode (ESI-). PLS-DA showed that the ASD and TD groups were well clustered with particular metabolic profiles for each (ESI+ : $\mathrm{R}^{2} \mathrm{Y}=0.73, \mathrm{Q}^{2}=0.61, p<0.0001$; ESI-: $\mathrm{R}^{2} \mathrm{Y}=0.76, \mathrm{Q}^{2}=0.65, p<0.0001$; Fig. 1a, b). The permutation test with $p<0.001$ indicated that the classification of global metabolite profiles between the ASD and TD groups was significantly different. We identified 96 differential metabolites between the ASD and TD groups, including 35 significantly increased metabolites and 61 significantly decreased metabolites in the ASD group (Table S1).

KEGG pathway analysis revealed 27 KEGG pathways associated with ASD status (Table S2). Interestingly, the differential metabolites were mainly enriched in multiple vitamin and amino acid metabolism pathways, with the strongest enrichment identified for tryptophan metabolism $(p=0.0006)$, retinol metabolism $(p=0.009)$, cysteine and methionine metabolism $(p=0.008)$, and vitamin digestion and absorption ( $p=0.01$; Fig. 1c). In addition, some differential metabolites were involved in arachidonic acid, steroid hormone, citrate cycle, and purine metabolism.

Disturbances in various vitamin metabolism pathways were found in children with ASD, as shown in Table 2 and Fig. 2. In the retinol metabolism pathway, abnormal levels of precursors and intermediates of vitamin A were found in the ASD group. The 4'-apo-beta-carotenal, b,ecarotene-3,3'-diol, and retinal levels were increased, while retinol level was decreased. The concentrations of multiple $B$ vitamins and their derivatives were decreased, including thiamine pyrophosphate (TPP), riboflavin (vitamin B2) and its intermediate lumichrome, phosphopantothenic acid (vitamin B5 derivative), pyridoxamine (vitamin B6), 1,4,5,6-tetrahydro-6-oxonicotinic acid, dihydrofolate (DHF), and 5-methyltetrahydrofolate(5-MTHF). The vitamin $\mathrm{C}$ level was also decreased in the children with ASD.

Aberrant amino acid metabolisms were also associated with ASD (Fig. 2), and tryptophan metabolism pathway was the most affected. The concentrations of xanthurenic acid, 5-hydroxy- $N$-formylkynurenine, 5-hydroxytryptophan (5-HTP), serotonin (5-hydroxytryptamine, 5-HT), and $N$-feruloyl serotonin were significantly increased in the ASD group, while the 6-hydroxymelatonin and 5-hydroxyindoleacetic acid (5-HIAA) levels were decreased in the ASD group. Besides, some indole derivatives, including indole acrylic acid and indole-2-carboxylic acid, were decreased in the ASD group. The cysteine-methionine metabolism pathway is closely related to folate metabolism. Both pathways showed abnormalities in the ASD group, with lower levels of DHF, 5-MTHF, $N$-acetylcysteine (NAC), and $S$-aminoethyl- $L$-cysteine, and excessive accumulation of homocysteine (Hcy). In the case of arginine metabolism pathway, the concentrations of polyamines, including agmatine, spermine, and glutathione spermidine, were lower in children with ASD. We also found abnormal glutamate and glycine 
a

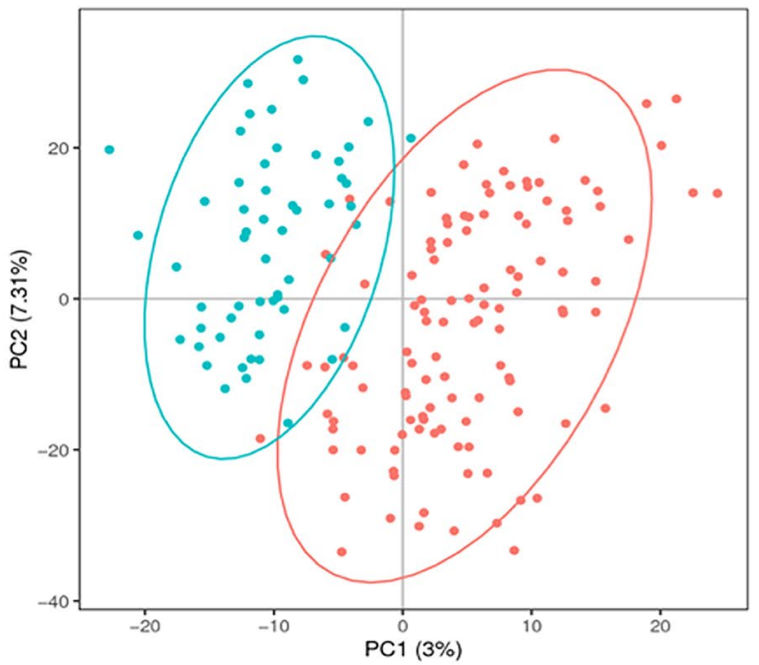

b

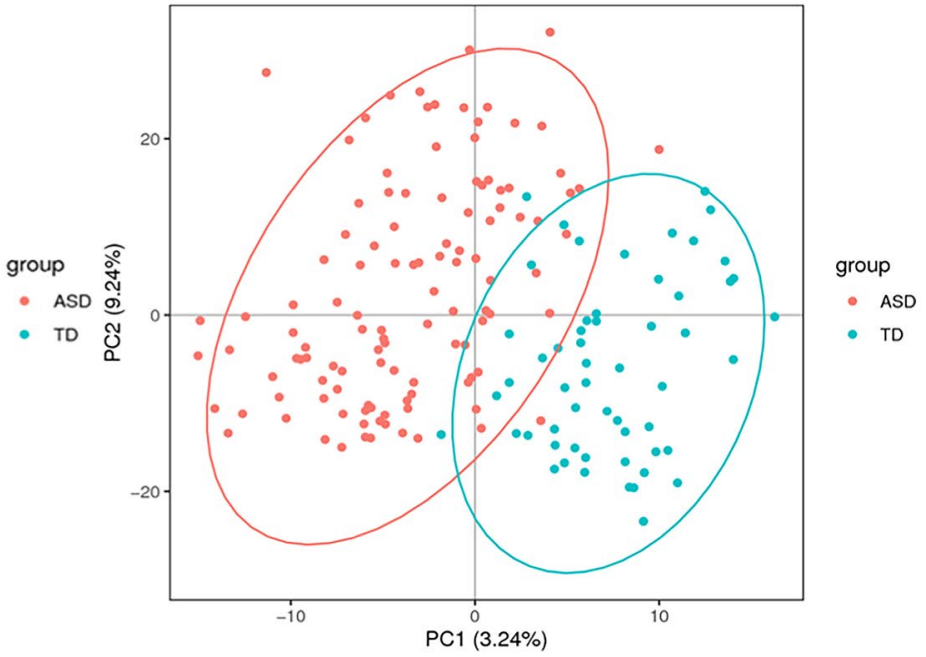

C

Pathway enrichment

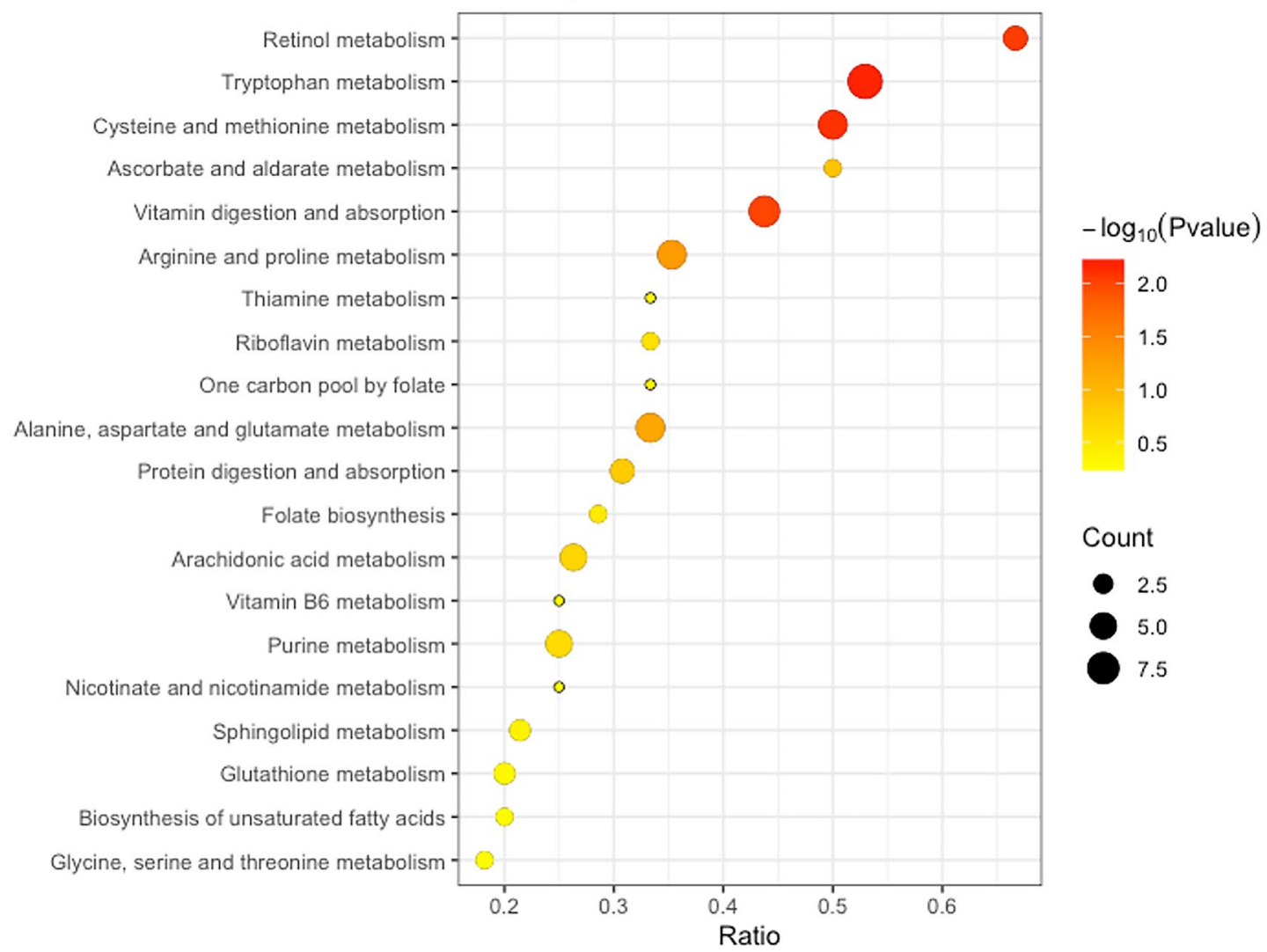

Fig. 1 Alterations of the gut metabolome in children with ASD compared with TD children. a, b The clustering analyses of partial leastsquares discriminant analysis (PLS-DA) of gut metabolome data in the positive ion mode (a) and negative ion mode (b). c Top 20 KEGG pathways enriched by differential gut metabolites between the ASD

metabolism, with decreased glutamine, GABA, and glycine levels in children with ASD. and TD children. Count, the number of differential metabolites in the pathway. Ratio, the ratio of number of differential metabolites to all detected metabolites in the pathway. $p$ value, $p$ value of hypergeometric test. $A S D$ autism spectrum disorders, $T D$ typically developing

Disturbances were also detected in the biologically active metabolites of arachidonic acid, which are crucial regulators of oxidative stress and inflammation. Arachidic 
Table 2 Aberrant gut metabolites relevant to vitamins and cofactors in children with ASD

\begin{tabular}{|c|c|c|c|c|}
\hline Vitamins metabolites & Metabolism pathway & Fold change $^{\mathrm{a}}$ & $p$ value & $\begin{array}{l}\text { Regula- } \\
\text { tion } \\
\text { mode }^{b}\end{array}$ \\
\hline 4'-Apo-beta-carotenal & Vitamin digestion and absorption, retinol metabolism & 1.55 & $9.24 \mathrm{E}-05$ & up \\
\hline b,e-Carotene-3,3'-diol & Vitamin digestion and absorption, retinol metabolism & 1.56 & 0.0043 & up \\
\hline All-trans-retinal & Retinol metabolism & 1.56 & 0.0262 & up \\
\hline Retinol & Retinol metabolism & 0.64 & 0.0149 & down \\
\hline Tocopherol & Vitamin E metabolism & 3.19 & 0.002 & up \\
\hline Thiamine pyrophosphate & Thiamine metabolism & 0.45 & 0.0224 & down \\
\hline Riboflavin tetrabutyrate & Riboflavin metabolism & 0.21 & 0.0085 & down \\
\hline$(+)$-Riboflavin & Riboflavin metabolism & 0.62 & 0.0305 & down \\
\hline Lumichrome & Riboflavin metabolism & 0.64 & 0.005 & down \\
\hline Pyridoxamine & Vitamin digestion and absorption, vitamin B6 metabolism & 0.63 & 0.0035 & down \\
\hline Phosphopantothenic acid & Vitamin B5 metabolism & 0.64 & 0.0181 & down \\
\hline 5-Methyltetrahydrofolate & Folate biosynthesis & 0.56 & 0.0068 & down \\
\hline Dihydrofolic acid & Vitamin digestion and absorption, folate biosynthesis & 0.49 & 0.006 & down \\
\hline $\begin{array}{l}\text { 1,4,5,6-Tetrahydro-6-oxoni- } \\
\text { cotinic acid }\end{array}$ & Nicotinate and nicotinamide metabolism & 0.66 & 0.0036 & down \\
\hline Vitamin C & Vitamin digestion and absorption, ascorbate and aldarate metabolism & 0.58 & 0.0148 & down \\
\hline L-Ascorbic acid & Ascorbate and aldarate metabolism & 0.51 & 0.0031 & down \\
\hline
\end{tabular}

${ }^{\mathbf{a b}}$ Fold change and regulation mode in the ASD group compared with the typically developing group $A S D$ autism spectrum disorders

Fig. 2 Metabolism pathway networks of the differential metabolites between the ASD and TD group. Gut metabolites with a fold change $>1.5$, a variable importance in projection score(VIP) $>1$, and a FDR-corrected $p$ values $<0.05$ for the Student's $t$ test or Mann-Whitney $U$ test were considered significantly differentially expressed between the two groups. Red font $(\uparrow)$, metabolites increased in the ASD group; Green font $(\downarrow)$, metabolites decreased in the ASD group; black font, no significant difference between the ASD and TD groups; grey font, undetected. DHF, dihydrofolate; 5-MTHF, 5-methyltrahydrofolate; SAM, S-adenosylmethionine; SAH, S-adenosylhomocysteine. $A S D$ autism spectrum disorders; $T D$ typically developing

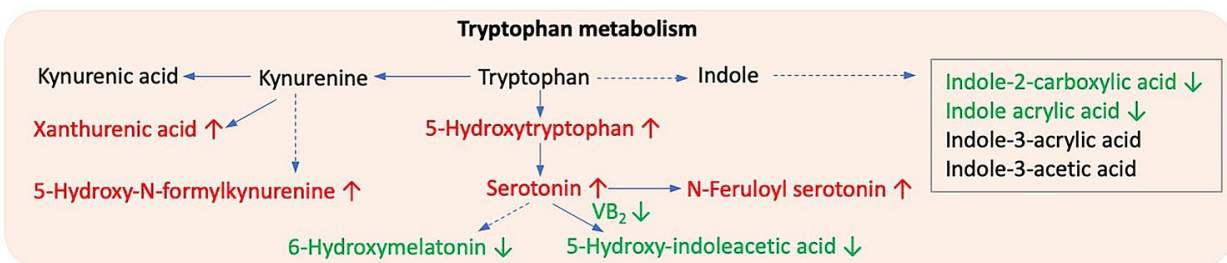

Cysteine and methionine metabolism,

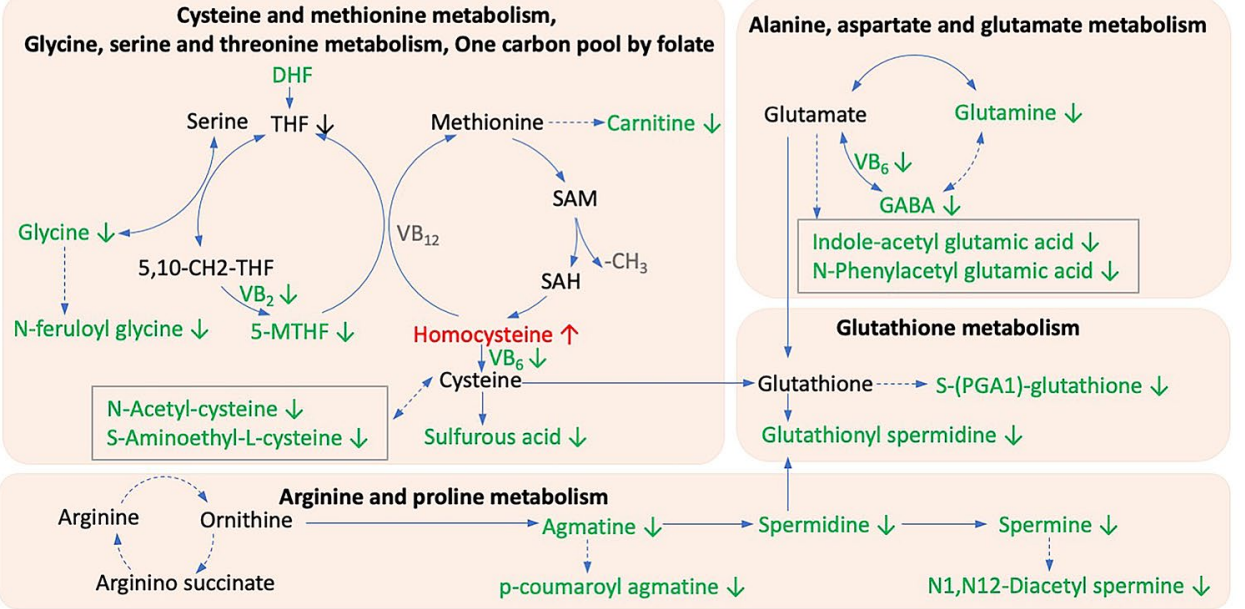

acid and 20-hydroxy-leukotriene E4 levels were increased, while leukotriene B4 and 5-trans prostaglandin F2 $\beta$ levels were decreased in children with ASD. Besides, the purine metabolite 8 -hydroxy-deoxyguanosine (8-OHdG), which is a sensitive marker of oxidative DNA damage (Valavanidis et al., 2009), was significantly increased (6.86-fold; $p=0.001)$ in the ASD group. 


\section{Correlation of Gut Metabolites with ASD Symptoms and Neurodevelopment Levels}

Spearman correlation analysis was performed to explore the potential links between key fecal metabolites and clinical assessment scores of children with ASD. Agmatine, $S$-aminoethyl- $L$-cysteine, 6-Hydroxymelatonin, pyridoxamine, GABA, and 5-trans prostaglandin F2 $\beta$ were negatively correlated with partial subscales or total ABC, SRS or CARS scores. Conversely, retinal, Hcy, serotonin, N-feruloyl serotonin, and 5-HIAA in the gut were positively correlated with ASD symptoms. $S$-aminoethyl- $L$-cysteine, 5 -trans prostaglandin F2 $\beta$, and retinol were positively correlated with neurodevelopment scores, while 8-OHdG, Hcy, 5-hydroxy$\mathrm{N}$-formylkynurenine, and serotonin were negatively correlated with neurodevelopment scores (Fig. 3; Table S3).

\section{Impact of GI Problems on the Metabolic Patterns in ASD Children}

Gastrointestinal (GI) problems is often co-occurred with ASD and we found $48.33 \%$ of ASD children suffered from GI symptoms. The impact of GI problems on the gut metabolic patterns of children with ASD was evaluated. The supervised PLS-DA showed that the ASD children with GI symptoms (ASD-GI, $n=58$ ) and non-GI symptoms (ASD-nonGI, $n=62$ ) were not partly clustered with specific metabolic profiles for each $\left(E S I+: \mathrm{R}^{2} \mathrm{Y}=0.27, \mathrm{Q}^{2}=0.10\right.$, $p=0.14$; ESI-: $\left.\mathrm{R}^{2} \mathrm{Y}=0.25, \mathrm{Q}^{2}=0.06, p=0.045\right)$, while the metabolic profiles of the both groups were different from the TD group (all $p$ value $<0.001$; Figure S1a-b). We found most differential metabolites revealed between ASD and TD groups were not different between ASD-GI and ASD-nonGI groups (data not shown). The results showed GI problems had a limited impact on the metabolic patterns and presence of differential metabolites.

Spearman correlation analysis revealed that serotonin and $\mathrm{N}$-feruloyl serotonin levels were positively correlated with 6-GSI scores ( $\mathrm{rs}=0.185, p=0.044$; $\mathrm{rs}=0.276, p=0.002$ ). We found a positive association between dihydrofolic acid levels and 6-GSI scores ( $\mathrm{rs}=0.341, p<0.001$ ), which indicated GI problems may affect the absorption of folic acid.

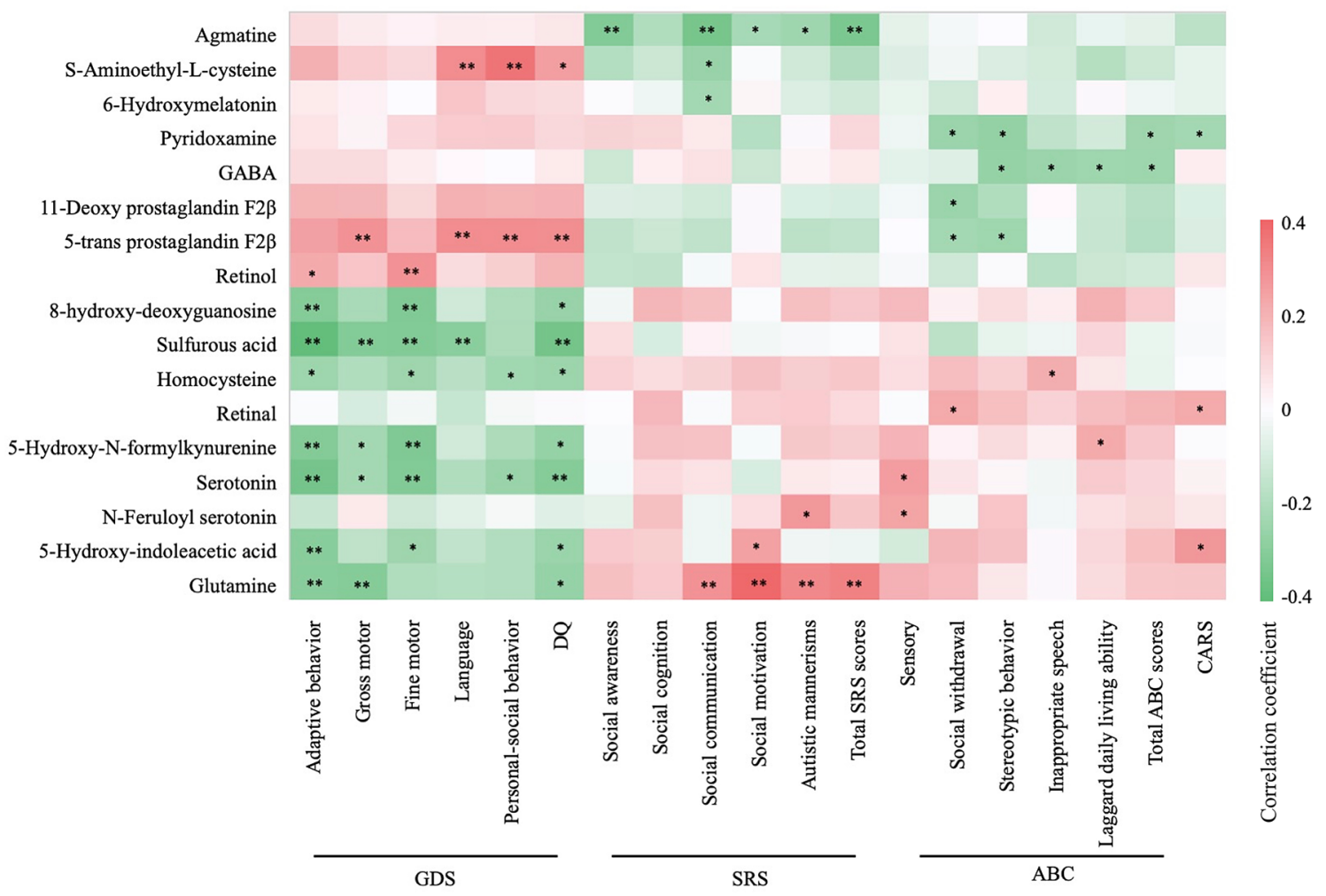

Fig. 3 Correlations of gut metabolites with ASD symptoms and neurodevelopment levels. The Spearman correlation coefficient is indicated by a color gradient from green (negative correlation) to red (positive correlation). The ${ }^{*}$ symbol in each lattice represent a sig- nificant correlation. ${ }^{*} p<0.05, * * p<0.01$. GDS Gesell Developmental Scale, $A B C$ autism behavior checklist, SRS Social Responsiveness Scale, $C A R S$ Childhood Autism Rating Scale, $D Q$ development quotient scores. 


\section{Discussion}

This study showed that gut metabolomic profiles significantly differed between young children with ASD and TD children. The differential fecal metabolites were mainly involved in vitamin and amino acid metabolism pathways, with the strongest enrichment identified for tryptophan metabolism, retinol metabolism, cysteine and methionine metabolism, and vitamin digestion and absorption. Some metabolic perturbations were associated with ASD symptoms and neurodevelopment levels, and may contribute to the pathogenesis of ASD through the gut-brain axis.

Vitamin A is required for functional systemic development in children (McLean et al., 2020), and studies have showed that children with ASD are more vulnerable than neurotypical children to vitamin A deficiency (Guo et al., 2018; Ranjan et al. 2015). In our study, the increased 4'-apo-beta-carotenal and b,e-carotene-3,3'-diol levels and decreased retinol level may indicate that children with ASD had a decreased capacity for the absorption and bioconversion of plant-origin precursors of vitamin A. Vitamin A has three active forms in humans: retinal, retinol, and retinoic acid (RA) (Kedishvili, 2016). RA, the main active form of vitamin $\mathrm{A}$, is a crucial signaling molecule that regulates multiple fundamental biological processes (Kedishvili, 2016). The increased retinal level in our study may imply that the conversion of retinal to RA was suppressed in the gut of children with ASD. Excessive retinal may damage the nervous system. We found that in children with ASD, the retinol level was positively correlated with neurodevelopment levels, and the retinal level was positively correlated with the social withdrawal subscale of SRS. The ALDH1A family consists of key enzymes that oxidize retinal into RA, and XX Xu et al. (Xu et al., 2018) found that ASD patients with excessive UBE3A (an autism-related gene and molecule) may have congenital errors of retinol metabolism, as excessive UBE3A can inhibit ALDH1A activity and compromise the oxidation of retinal to RA. Moreover, the gut microbiota can participate in the alternative biotransformation of retinal to retinol or RA (Hong et al., 2016).

$\mathrm{B}$ vitamins are important cofactors implicated in multiple biochemical reactions. TPP, a derivative of thiamine (vitamin B1), is a cofactor of various enzymes in the mitochondria. Anwar A et al. (Anwar et al., 2016) found that plasma TPP concentrations were significantly lower in children with ASD than in controls. Consistent with this, we found lower levels of TPP in the feces of children with ASD than in TD children. Decreased TPP can lead to reduced mitochondrial anti-oxidative potential and energy production, and subsequently cellular damage (Altuner et al., 2013; Cinici et al., 2018). Vitamins B2 and B6 also participate in multiple amino acids metabolism processes. We found the level of pyridoxamine, a form of vitamin B6, was slightly negatively correlated to $\mathrm{ABC}$ and CARS scores.

The pathways of cysteine and methionine cycle, folate(vitamin B9) metabolism, and Hcy transsulfuration are interrelated and together constitute the folate-related metabolism pathway (Zou et al., 2019), which is critical for cell proliferation, DNA synthesis, immune function, and neural development (Sun et al., 2016). Vitamins B6 and B12 are cofactors in these biological processes. Decreased folate and vitamin B6 levels may lead to Hcy accumulation and decreased methyl production. Much of evidence suggested that folate deficit and excessive Hcy are risk factors for neural tube defects and neurodevelopmental disorders (Türksoy et al., 2014), and children with ASD have decreased folate levels and elevated Hcy levels in the blood and urine (Paşca et al., 2006; Yektaş et al., 2019). In our study, Hcy levels were negatively correlated with neurodevelopment scores, indicating the adverse impact of excessive Hcy on brain development and function. Moreover, NAC is an antioxidant with potential benefits in treating the irritability in children with ASD (Nikoo et al., 2015).

Abnormal tryptophan metabolism pathway in ASD has been reported in multiple studies, which was characterized by decreased tryptophan concentrations (Ormstad et al., 2018) and increased serotonin levels in the blood (Muller et al., 2016). In the gut, there are three main tryptophan metabolism pathways, which lead to kynurenine, serotonin, and indole derivatives (Agus et al., 2018; KałużnaCzaplińska et al., 2019). Through the kynurenine pathway, kynurenic acid, xanthurenic acid, and quinolinic acid are generated (Agus et al., 2018). In our study, xanthurenic acid and 5-hydroxy- $N$-formylkynurenine levels were significantly increased in the ASD group. Vitamin B6 is a cofactor of kynureninase and kynurenine aminotransferase; therefore, the decrease of B6 may have contributed to the increased xanthurenic acid and 5-hydroxy- $N$-formylkynurenine levels. In the serotonin pathway, 5-HTP, serotonin, and $N$-feruloyl serotonin were significantly increased in feces of children with ASD, while 6-hydroxymelatonin and 5-HIAA were decreased. Reproducible evidence suggested serotonin-melatonin pathway in ASD is impaired, leading to hyperserotonemia and melatonin deficit in plasma (Abdulamir et al., 2018; Pang et al., 2014; Muller et al., 2016). However, few studies have reported altered tryptophan metabolism and serotoninmelatonin levels in the gut of ASD patients. Angelis et al. (De Angelis et al., 2013) found increased tryptophan and 3-methylindole levels in the feces of children with ASD. Dan $\mathrm{Z}$ et al. (Dan et al., 2020) also reported abnormal tryptophan metabolism in the gut of children with ASD. An mice model of autism found decreased serotonin in intestine mucosal (Golubeva et al., 2017). However, given that $95 \%$ of the serotonin in the body is generated in the intestine (Colle et al., 2020), it is likely that blood serotonin levels are correlated 
with enteric serotonin. Likewise, the GI tract, in addition to the pineal gland, is an important source of melatonin besides the pineal gland (Gagnon \& Godbout, 2018). Melatonin can regulate sleep patterns, immunity, as well as GI function (Gagnon \& Godbout, 2018). Serotonin can be catabolized to 5-HIAA, and this process depends on riboflavin (vitamin B2) as a cofactor, so riboflavin deficiency may be related to the increase of serotonin. Moreover, dysbiosis of the gut microbiota has been linked to abnormal tryptophan metabolism (Agus et al., 2018). We found a negative correlation between gut serotonin levels and neurodevelopment scores of ASD children, while serotonin and $N$-feruloyl serotonin levels were positively correlated with the sensory subscales of $\mathrm{ABC}$ and GI problems. Many studies have indicated that the blood serotonin levels are correlated with the severity of autism severity (Abdulamir et al., 2018). A balanced amount of enteric serotonin is beneficial to the functioning of the intestine, nervous system, and gut-brain axis, while excess serotonin may play a harmful role in the ASD progression.

We found decreased GABA, glutamine, glycine, and polyamines in fecal of children with ASD. GABA was negatively correlated with $\mathrm{ABC}$ scores, and agmatine was negatively correlated with SRS scores. These amino derivatives are crucial neurotransmitters or neuromodulators in the nervous system, and are important for immunity and inflammation (Ueland et al., 2017). GABA and glycine are inhibitory neurotransmitters, and their decrease may impact the excitation-inhibition balance of the nervous system (Nelson \& Valakh, 2015). Our findings are partially supported by Kang DW et al. 2018 and Angelis et al. (De Angelis et al., 2013), who reported possibly lower GABA concentrations in the gut of children with ASD compared with healthy controls. Ford et al. (2020) found that aberrant glutamate and GABA processes were linked with impaired psychosocial function. Particularly, the synthesis of both GABA and glycine depend on vitamin B6 as a cofactor (Sato, 2018).

Biologically active metabolites of arachidonic acid showed disturbance, which are key regulators in oxidative stress and inflammation (Sergeant et al., 2016). Besides, 8-OHdG, a purine metabolite, is a sensitive marker of oxidative DNA damage (Valavanidis et al., 2009). Elevated 8-OHdG levels has been found in the cerebellar (SajdelSulkowska et al., 2009) and urinary excretion (Ming et al., 2005) of ASD patients. In the present study, 8-OHdG was significantly increased (6.86-fold) in autistic children compared to TD children. These results indicates that children with ASD may have a higher risk of gastrointestinal damage by oxidative stress and inflammation.

Gut metabolism is the result of interactions of multiple genetic and environmental factors, including disease, microbiome, and diet (Alexander \& Turnbaugh, 2020). Gut microbiota are important for gut metabolism, as microflora can produce vitamins and participate in the metabolism of numerous substances (Hong et al., 2016; LeBlanc et al., 2013). Picky eating is almost one of important characterizations of children with ASD, so inadequate intake from food could also partly explain the decreased in multiple vitamins and amino acids. In addition, GI problems may affect the absorption of nutrients. Furthermore, vitamin abnormalities/ deficiencies may contribute to altered amino acid metabolism, for vitamins B are implicated in multiple biochemical reactions (Sato, 2018). Metabolic interventions for ASD include supplementation of prebiotics and probiotics, vitamins (e.g., A, C, D, B6, B12, folate), amino acids, and their derivatives (e.g., glycine, NAC) (Bjørklund et al. 2019; DeFilippis, 2018; Höfer et al., 2017; Mierau \& Neumeyer, 2019). These approaches could sometimes correct intestinal dysbiosis and nutritional deficiencies in ASD, and partly improve the downstream metabolic consequences. However, these interventions were not always effective (Bjørklund et al., 2019), for some inborn errors of metabolism are hard to rectify, and single-compound supplementation may be insufficient to overcome the extensive abnormalities of metabolic networks in ASD. Therefore, detailed evaluation and individualized interventions for ASD children are required.

\section{Limitations}

There are limitations in the present study. First, this crosssectional study revealed correlations, but our data do not allow to prove the causation of symptoms and gut metabolites outcome. In addition, the correlations were not very strong (correlation coefficients, $0.2-0.4$ ), as the metabolic disturbance is only one of many factors related to neurological function and ASD symptoms. Second, fecal metabolism may reflect the final results of the interactions of diet, microbiota, and intestinal function; however, the metabolic activity of each intestinal segment and the absorption and utilization of metabolites remain unclear, and it was difficult to distinguish whether the metabolites are derived from the host or the gut microbiota. Thus, the simultaneous analysis of fecal, intestinal contents, blood, microbiota, and other biological samples may lead to a deeper understanding of metabolomics networks. Third, our participants were preschool children from an island of China with a comparably biological backgrounds; therefore, these findings may not be generalizable to all ASD patients in different regions, races, and ages. Finally, ASD is a group of complex neurodevelopmental disorders, and studies involving different ASD subtypes and other related diseases are needed to evaluate the disease specificity of the metabolomic disturbances (age, sex, with our without food selectivity, developmental delay, 
etc.). Some metabolic disturbances may be nonspecific for various neurodevelopmental diseases and have an extensive impact on brain function and neurodevelopment.

\section{Conclusions}

Children with ASD exhibit gut-metabolism perturbations that mainly involve amino acid and vitamin metabolism. These perturbations are related to ASD symptoms and neurodevelopment levels, and may be the result of the interaction of multiple factors, including congenital metabolism errors, decreased intake due to abnormal eating patterns, and intestinal microflora imbalance (Fig. 4). Notably, in the interrelated metabolism networks, vitamin metabolism abnormalities and decreased vitamin intake may disturb the amino acid metabolism, as B vitamins are essential cofactors implicated in multiple biochemical reactions. The differential metabolites may affect the brain development and function, and subsequently behavior via nutrition, neurotransmitter, immune-inflammation modulatory, and other pathways. Approaches such as nutritional supplementation and regulation of the intestinal flora may partially benefit the gut metabolism, nutritional status, and symptoms in ASD. It is essential to perform a detailed evaluation and provide comprehensive and individualized interventions for children with ASD. Our findings provided an extensive understanding of the disturbances in metabolism networks in ASD.

Supplementary Information The online version contains supplementary material available at https://doi.org/10.1007/s10803-021-05066-w.

Acknowledgments Not applicable.

Author Contributions JZ, JC, and TL were involved in designing the trial and writing the trial protocol, analyzing the data and writing the manuscript. JC, LL and TL were also involved in clinical assessment, supervising subjects' recruitment, data collection and drafting the manuscript. JZ, XH, TY, MG, QL, and LX were involved in experimental operation, data collection, analyzing the data and revising the manuscript. JC, and TL had primary responsibility for final content. All authors approved the final version of the manuscript.

Funding This study is funded by the National Natural Science Foundation of China (No. 81771223; 81770526) (http://www.nsfc.gov.cn), and the Guangdong Key Project in Development of new tools for diagnosis and treatment of autism (2018B030335001), and the Guangzhou Key Project in Early diagnosis and treatment of autism spectrum disorders (202007030002).

Data Availability All data generated and analyzed in the current study are available from the corresponding author on reasonable request.
Fig. 4 Hypothesis of interplay between the gut metabolism and the gut-brain axis in ASD. Aberrant of gut metabolism profiles in ASD may be the result of the interactions of multiple factors, including congenital errors in metabolism, decreased intake due to abnormal eating patterns, and intestinal microflora imbalance. In the interrelated metabolism networks, vitamin metabolism abnormalities and decreased vitamin intake may disturb the amino acid metabolism, as vitamins $B$ are essential cofactors implicated in multiple biochemical reactions. The altered metabolites may affect the brain development and function, and subsequently behavior by nutrition, neurotransmitters, immune-inflammation modulatory, and other pathways

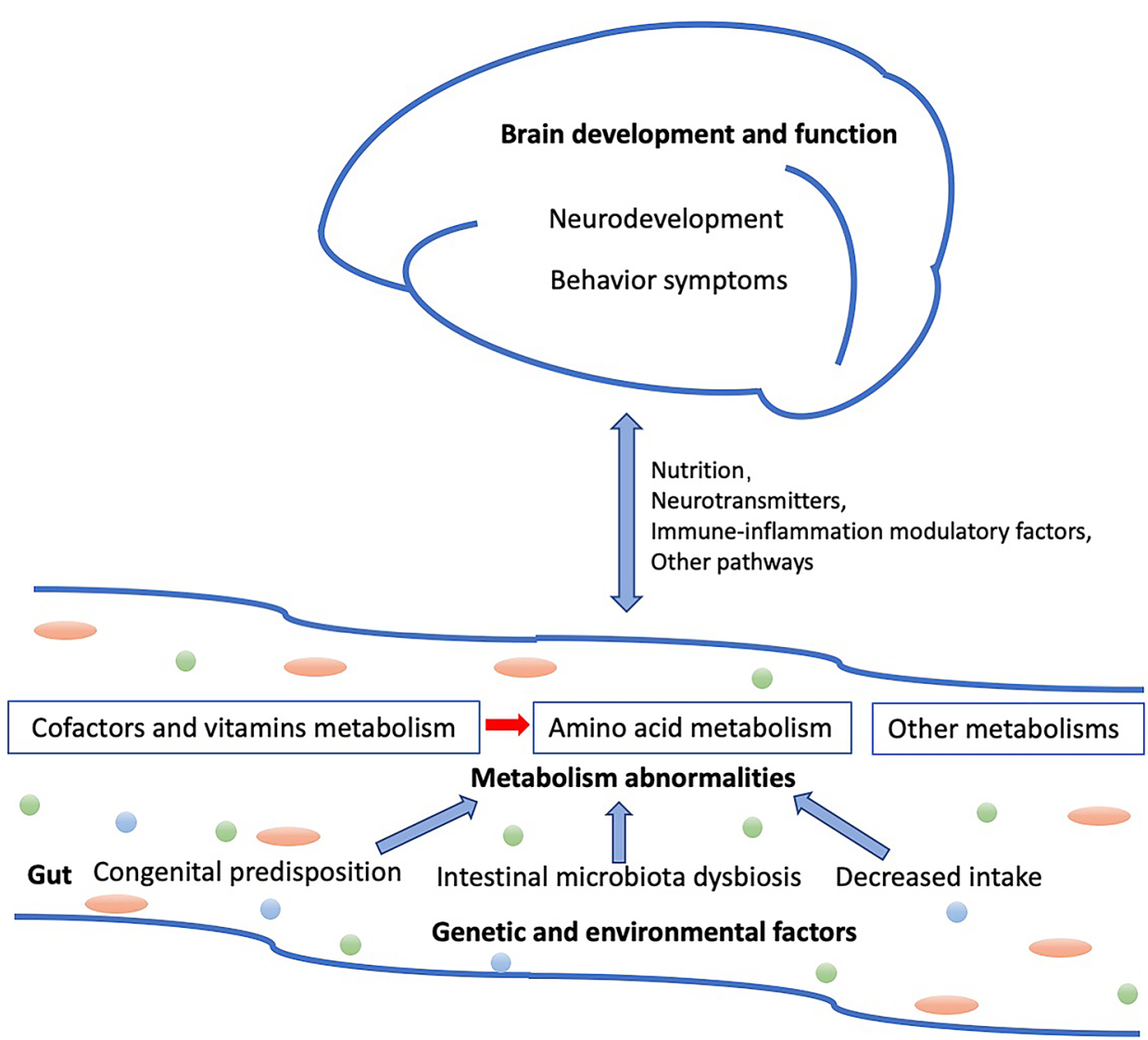




\section{Declarations}

Conflict of interests The authors declare that they have no competing interests.

Ethics Approval and Consent to Participate Participation in this research was voluntary. Parents signed written informed consent forms and were willing to let their children participate in the study. The study protocol was approved by the institutional review board of Children's Hospital, Chongqing Medical University. This cross-sectional casecontrol study was based on a clinical trial which was registered in the Chinese Clinical Trial Registry (ChiCTR; registration number: ChiCTR-ROC-14005442).

\section{Consent for Publication Not applicable.}

Open Access This article is licensed under a Creative Commons Attribution 4.0 International License, which permits use, sharing, adaptation, distribution and reproduction in any medium or format, as long as you give appropriate credit to the original author(s) and the source, provide a link to the Creative Commons licence, and indicate if changes were made. The images or other third party material in this article are included in the article's Creative Commons licence, unless indicated otherwise in a credit line to the material. If material is not included in the article's Creative Commons licence and your intended use is not permitted by statutory regulation or exceeds the permitted use, you will need to obtain permission directly from the copyright holder. To view a copy of this licence, visit http://creativecommons.org/licenses/by/4.0/.

\section{Reference}

Abdulamir HA, Abdul-Rasheed OF, Abdulghani EA. (2018). Serotonin and serotonin transporter levels in autistic children. Saudi Medical Journal, Retrieved August 13, 2020, from: http://www.ncbi.nlm. nih.gov/pubmed/29738009

Agus A, Planchais J, Sokol H. Gut Microbiota. (2018). Regulation of tryptophan metabolism in health and disease. Cell Host Microbe, 23(6):716-24. Retrieved August 13, 2020, from: http://www.ncbi. nlm.nih.gov/pubmed/29902437

Alexander M, Turnbaugh PJ. (2020). Deconstructing mechanisms of diet-microbiome-immune interactions. Immunity, 53(2):264-76. August 25, 2020, from: http://www.ncbi.nlm.nih.gov/pubmed/ 32814025

Altuner D, Cetin N, Suleyman B, Aslan Z, Hacimuftuoglu A, Gulaboglu M, Isaoglu, N., Demiryilmaz, I. and Suleyman, H. (2016). Effect of thiamine pyrophosphate on ischemia-reperfusion induced oxidative damage in rat kidney. Indian Journal of Pharmacology, 45(4):339-43. Retrieved August 12, 2020, from: http:// www.ncbi.nlm.nih.gov/pubmed/24014907

American Psychiatric Association. (2013). Diagnostic and Statistical Manual of Mental Disorders. (5th ed.). American Psychiatric Association Publishing.

Anwar A, Marini M, Abruzzo PM, Bolotta A, Ghezzo A, Visconti P Thornalley, P.J. and Rabbani, N. (2016). Quantitation of plasma thiamine, related metabolites and plasma protein oxidative damage markers in children with autism spectrum disorder and healthy controls. Free radical research, 50(sup1):S85-90. Retrieved August 12, 2020, from: http://www.ncbi.nlm.nih.gov/pubmed/ 27667096

Bjørklund G, Waly MI, Al-Farsi Y, Saad K, Dadar M, Rahman MM, Elhoufey, A., Chirumbolo, S., Jóźwik-Pruska, J. and KałużnaCzaplińska, J. (2019). The Role of vitamins in autism spectrum disorder: What do we know? Journal of Molecular Neuroscience, 67(3):373-87. Retrieved August 13, 2020, from: http://link. springer.com/https://doi.org/10.1007/s12031-018-1237-5

Cen, C-Q., Liang, Y-Y., Chen, Q-R., Chen, K-Y., Deng, H-Z., Chen, B-Y., Zou, X.B. (2017) Investigating the validation of the Chinese Mandarin version of the Social Responsiveness Scale in a Mainland China child population. BMC Psychiatry, 17(1):51. Retrieved January 13, 2019, from: http://www.ncbi.nlm.nih.gov/ pubmed/28166747

Cinici, E., Mammadov, R., Findik, H., Suleyman, B., Cetin, N., Calik, I., Balta, H., Hakki Tas, I., Sener, E., \& Altuner, D. (2018). The protective effect of thiamine pryophosphate against sugar-induced retinal neovascularisation in rats. International Journal for Vitamin and Nutrition, 88(3-4), 137-43

Chang P V, Hao L, Offermanns S, Medzhitov R. (2014). The microbial metabolite butyrate regulates intestinal macrophage function via histone deacetylase inhibition. Proceedings of the National Academy of Sciences, 111(6):2247-52. Retrieved February 6, 2020, Available from: http://www.pnas.org/lookup/doi/https://doi.org/ 10.1073/pnas.1322269111

Dan, Z., Mao, X., Liu, Q., Guo, M., Zhuang, Y., Liu, Z., Chen, K., Chen, J., Xu, R., Tang, J., \& Qin, L. (2020). Altered gut microbial profile is associated with abnormal metabolism activity of autism spectrum disorder. Gut Microbes, 11(5), 1246-67. https://doi.org/ 10.1080/19490976.2020.1747329

De Angelis, M., Piccolo, M., Vannini, L., Siragusa, S., De Giacomo, A., Serrazzanetti, D.I., Cristofori, F., Guerzoni, M.E., Gobbetti, M. and Francavilla, R., (2013). Fecal microbiota and metabolome of children with autism and pervasive developmental disorder not otherwise specified. PLoS One, 8(10). Retrieved August 12, 2020, from: https://pubmed.ncbi.nlm.nih.gov/24130822/

Del Colle A, Israelyan N, Gross Margolis K. (2020). Novel aspects of enteric serotonergic signaling in health and brain-gut disease. American Journal of Physiology-Gastrointestinal and Liver Physiology, 318(1):G130-43. Retrieved August 13, 2020, from: http:// www.ncbi.nlm.nih.gov/pubmed/31682158

DeFilippis M. (2018). The use of complementary alternative medicine in children and adolescents with autism spectrum disorder. Psychopharmacology Bulletin, 48(1):40-63. Retrieved August 13, 2020, from: http://www.ncbi.nlm.nih.gov/pubmed/29382959

Ford TC, Woods W, Enticott PG, Crewther DP. (2020). Cortical excitation-inhibition ratio mediates the effect of pre-attentive auditory processing deficits on interpersonal difficulties. Progress in Neuro-Psychopharmacology and Biological Psychiatry, 98:109769. Retrieved August 13, 2020, from: http://www.ncbi. nlm.nih.gov/pubmed/31676468

Gagnon K, Godbout R. (2018). Melatonin and Comorbidities in Children with Autism Spectrum Disorder. Current Developmental Disorders Reports, 5(3):197-206. Retrieved August 13, 2020, from: http://www.ncbi.nlm.nih.gov/pubmed/30148039

Glinton KE, Elsea SH. (2019). Untargeted metabolomics for autism spectrum disorders: Current status and future directions. Frontiers in Psychiatry, 10. Retrieved August 11, 2020, from: https://www. ncbi.nlm.nih.gov/pmc/articles/PMC6746843/

Golubeva A V, Joyce SA, Moloney G, Burokas A, Sherwin E, Arboleya S, Flynn, I., Khochanskiy, D., Moya-Pérez, A., Peterson, V. and Rea, K. (2017). Microbiota-related changes in bile acid \& tryptophan metabolism are associated with gastrointestinal dysfunction in a mouse model of autism. EBioMedicine, 24:166-78. Reterived August 13, 2020, from: http://www.ncbi.nlm.nih.gov/ pubmed/28965876

Guo, M., Zhu, J., Yang, T., Lai, X., Lei, Y., Chen, J., \& Li, T. (2018). Vitamin A and vitamin D deficiencies exacerbate symptoms in children with autism spectrum disorders. Nutritional Neuroscience. https://doi.org/10.1080/1028415X.2017.1423268 
Höfer J, Hoffmann F, Bachmann C. (2017). Use of complementary and alternative medicine in children and adolescents with autism spectrum disorder: A systematic review. Autism, 21(4):387-402. Retrieved August 13, 2020, from: http://journals.sagepub.com/ doi/https://doi.org/10.1177/1362361316646559

Hong S-H, Ngo H-P-T, Nam H-K, Kim K-R, Kang L-W, Oh D-K. (2016). Alternative biotransformation of retinal to retinoic acid or retinol by an aldehyde dehydrogenase from Bacillus cereus. Applied and Environmental Microbiology, 82(13): 3940-3946. Retrieved August 12, 2020 from: http://www.ncbi.nlm.nih.gov/ pubmed/27107124

Hyman SL, Levy SE, Myers SM. (2020). Council on children with disabilities, section on developmental and behavioral pediatrics. Identification, evaluation, and management of children with autism spectrum disorder. Pediatrics, 145(1):e2019344. Retrieved February 2, 2020, from: http://www.ncbi.nlm.nih.gov/pubmed/ 31843864

Jin X, Sun Y, Jiang F, Ma J, Morgan C, Shen X. (2007) Care for development intervention in rural China: A prospective follow-up study. Journal of Developmental \& Behavioral Pediatrics, 28(3):213-8. Retrieved January 13, 2019, from: http://www.ncbi.nlm.nih.gov/ pubmed/17565288

Kałużna-Czaplińska J, Gątarek P, Chirumbolo S, Chartrand MS, Bjørklund G. (2019). How important is tryptophan in human health? Critical Reviews in Food Science and Nutrition, 59(1):7288. Retrieved August 12 2020, from: http://www.ncbi.nlm.nih. gov/pubmed/28799778

Kang D-W, Ilhan ZE, Isern NG, Hoyt DW, Howsmon DP, Shaffer M, Catherine A., Lozupone, Juergen Hahn, James B. Adams, and Rosa Krajmalnik-Brown (2018) Differences in fecal microbial metabolites and microbiota of children with autism spectrum disorders. Anaerobe, 49:121-31. Retrieved February 7, 2020, from: https://linkinghub.elsevier.com/retrieve/pii/S1075996417302305

Kedishvili NY. (2016) Retinoic Acid Synthesis and Degradation. Subcell Biochem, 81:127-161. Retrieved August 12, 2020, from: http://www.ncbi.nlm.nih.gov/pubmed/27830503

LeBlanc JG, Milani C, de Giori GS, Sesma F, van Sinderen D, Ventura M. (2013.) Bacteria as vitamin suppliers to their host: a gut microbiota perspective. Current Opinion in Biotechnology, 24(2):160 8. Retrieved August 13, 2020, from: http://www.ncbi.nlm.nih.gov/ pubmed/22940212

Liu S, Li E, Sun Z, Fu D, Duan G, Jiang M, Yu Y. (2019). Altered gut microbiota and short chain fatty acids in Chinese children with autism spectrum disorder. Scientific Report, 9(1):287. Retrieved February 14, 2020, from: http://www.nature.com/artic les/s41598-018-36430-Z

McLean E, Klemm R, Subramaniam H, Greig A. (2020) Refocusing vitamin A supplementation programmes to reach the most vulnerable. BMJ Global Health, 5(7). Retrieved August 12, 2020 from: http://www.ncbi.nlm.nih.gov/pubmed/32718947

Mierau SB, Neumeyer AM. (2019). Metabolic interventions in autism spectrum disorder. Neurobiology of Disease, 132:104544. Retrieved August 13, 2020, from: http://www.ncbi.nlm.nih.gov/ pubmed/31351171

Ming X, Stein TP, Brimacombe M, Johnson WG, Lambert GH, Wagner GC. (2005). Increased excretion of a lipid peroxidation biomarker in autism. Prostaglandins, Leukotrienes \& Essential Fatty Acids, 73(5):379-84. Retrieved August 13, 2020, from: http://www.ncbi. nlm.nih.gov/pubmed/16081262

Mohamadkhani A. (2018). Gut microbiota and fecal metabolome perturbation in children with autism spectrum disorder. Middle East Journal of Digestive Diseases, 10(4):205-12. Retrieved February 7, 2020, from: http://mejdd.org/index.php/mejdd/article/view/ 2039

Muhle RA, Reed HE, Stratigos KA, Veenstra-VanderWeele J. (2018). The emerging clinical neuroscience of autism spectrum disorder.
JAMA Psychiatry, 75(5):514. Retrieved February 2, 2020, from: http://www.ncbi.nlm.nih.gov/pubmed/29590280

Muller CL, Anacker AMJ, Veenstra-VanderWeele J. (2016). The serotonin system in autism spectrum disorder: From biomarker to animal models. Neuroscience, 321:24-41. Retrieved August 12, 2020, from: http://www.ncbi.nlm.nih.gov/pubmed/26577932

Nelson SB, Valakh V. (2015). Excitatory/Inhibitory Balance and Circuit Homeostasis in Autism Spectrum Disorders. Neuron, 87(4):684-98. Reterived August 13, 2020, from: http://www.ncbi. nlm.nih.gov/pubmed/26291155

Nikoo M, Radnia H, Farokhnia M, Mohammadi M-R, Akhondzadeh S. (2015). N-acetylcysteine as an adjunctive therapy to risperidone for treatment of irritability in autism: a randomized, double-blind, placebo-controlled clinical trial of efficacy and safety. Clin Neuropharmacology, 38(1):11-7. Retrieved August 13, 2020, from: http://www.ncbi.nlm.nih.gov/pubmed/25580916

Ormstad H, Bryn V, Verkerk R, Skjeldal OH, Halvorsen B, Saugstad OD, Isaksen, J. and Maes, M. (2018). Serum tryptophan, tryptophan catabolites and brain-derived neurotrophic factor in subgroups of youngsters with autism spectrum disorders. CNS Neurological Disorders Drug Targets, 17(8):626-39. Retrieved August 13, 2020, from: http://www.ncbi.nlm.nih.gov/pubmed/30033880

Pagan, C., Delorme, R., Callebert, J., Goubran-Botros, H., Amsellem, F., Drouot, X., Boudebesse, C., Le Dudal, K., Ngo-Nguyen, N., Laouamri, H. and Gillberg, C. (American Psychiatric Association). The serotonin- $\mathrm{N}$-acetylserotonin-melatonin pathway as a biomarker for autism spectrum disorders. Translational Psychiatry, 4(11). Retreived August 13, 2020, from: https://pubmed.ncbi. nlm.nih.gov/25386956/

Paşca SP, Nemeş B, Vlase L, Gagyi CE, Dronca E, Miu AC, Dronca, M. (2006).. High levels of homocysteine and low serum paraoxonase 1 arylesterase activity in children with autism. Life Sciences, 78(19):2244-8. Retrieved August 12, 2020, from: http://www.ncbi.nlm.nih.gov/pubmed/16297937

Ranjan, S., \& Nasser, J. A. (2015). Nutritional status of individuals with autism spectrum disorders : Do we know enough? Advances in Nutrition, 5(6), 397-407

Rellini E, Tortolani D, Trillo S, Carbone S, Montecchi F. (2004). Childhood Autism Rating Scale (CARS) and Autism Behavior Checklist (ABC) correspondence and conflicts with DSM-IV criteria in diagnosis of autism. Journal of Autism and Developmental Disorders, 34(6):703-8. Retrieved January 12, 2019, from: http://www.ncbi.nlm.nih.gov/pubmed/15679189

Sajdel-Sulkowska EM, Xu M, Koibuchi N. (2009). Increase in cerebellar neurotrophin-3 and oxidative stress markers in autism. Cerebellum, 8(3):366-72. Retrieved August 13, 2020, from: http://www.ncbi.nlm.nih.gov/pubmed/19357934

Sato K. (2018). Why is vitamin B6 effective in alleviating the symptoms of autism? Medical Hypotheses, 115:103-6. Retrieved August 13, 2020, from: http://www.ncbi.nlm.nih.gov/pubmed/ 29685187

Sergeant S, Rahbar E, Chilton FH. (2016). Gamma-linolenic acid, dihommo-gamma linolenic, eicosanoids and inflammatory processes. European Journal Pharmacology, 785:77-86. Retrieved August 13,2020, from: http://www.ncbi.nlm.nih.gov/pubmed/ 27083549

Shen L, Liu X, Zhang H, Lin J, Feng C, Iqbal J. (2020). Biomarkers in autism spectrum disorders: Current progress. Clinica Chimica Acta, 502:41-54. Retrieved August 11, 2020, from: https:// www.sciencedirect.com/science/article/pii/S00098981193217 83?via\%3Dihub

Sun C, Zou M, Zhao D, Xia W, Wu L. (2016). Efficacy of folic acid supplementation in autistic children participating in structured teaching: An open-label trial. Nutrients, 8(6). Retrieved August 13, 2020, from: http://www.ncbi.nlm.nih.gov/pubmed/27338456 
Thomas RH, Meeking MM, Mepham JR, Tichenoff L, Possmayer F, Liu S, MacFabe, D.F. (2012). The enteric bacterial metabolite propionic acid alters brain and plasma phospholipid molecular species: further development of a rodent model of autism spectrum disorders. Journal of Neuroinflammation, 9(1):153. Retrieved February 7, 2020, from: http://jneuroinflammation.biomedcentral.com/articles/https://doi.org/10.1186/ 1742-2094-9-153

Türksoy N, Bilici R, Yalçıner A, Ozdemir YÖ, Ornek I, Tufan AE, Kara, A. Kara, A. (2014). Vitamin B12, folate, and homocysteine levels in patients with obsessive-compulsive disorder. Neuropsychiatric disease and treatment, 10:1671-1675. Retrieved August 12, 2020, from: http://www.ncbi.nlm.nih.gov/pubmed/25228807

Ueland, P.M., McCann, A., Midttun, Ø. and Ulvik, A. (2017). Inflammation, vitamin B6 and related pathways. Molecular Aspects of Medicine, 53.Reterieved August 13, 2020, from: https://pubmed. ncbi.nlm.nih.gov/27593095/

Valavanidis A, Vlachogianni T, Fiotakis C. (2009) 8-hydroxy-2' -deoxyguanosine (8-OHdG): A critical biomarker of oxidative stress and carcinogenesis. Journal of environmental science and health Part C Environmental Carcinogenesis and Ecotoxicology Reviews, 27(2):120-39. Retrieved August 12, 2020, from: http:// www.ncbi.nlm.nih.gov/pubmed/19412858

Wang M, Wan J, Rong H, He F, Wang H, Zhou J, Cai, C., Wang, Y., Xu, R., Yin, Z. and Zhou, W. (2019). Alterations in gut glutamate metabolism associated with changes in gut microbiota composition in children with autism spectrum disorder. Manichanh C. (Eds.) mSystems, 4(1):e00321-18. Retrieved February 16, 2020, from: http://msystems.asm.org/lookup/doi/https://doi. org/10.1128/mSystems.00321-18

Xu X, Li C, Gao X, Xia K, Guo H, Li Y, Hao, Z., Zhang, L., Gao, D., Xu, C. and Xu, H. (2018). Excessive UBE3A dosage impairs retinoic acid signaling and synaptic plasticity in autism spectrum disorders. Cell Research, 28(1):48-68. Retrieved August 12, 2020, from: http://www.ncbi.nlm.nih.gov/pubmed/29076503

Yektaş, Ç., Alpay, M., \& Tufan, A. E. (2019). Comparison of serum B12, folate and homocysteine concentrations in children with autism spectrum disorder or attention deficit hyperactivity disorder and healthy controls. Neuropsychiatric Disease and Treatment, 15, 2213-2219

Zou M, Sun C, Liang S, Sun Y, Li D, Li L, Fan, L., Wu, L. and Xia, W., (2019). Fisher discriminant analysis for classification of autism spectrum disorders based on folate-related metabolism markers. The Journal of nutritional biochemistry, 64:25-31. Retrieved August 12, 2020, from: http://www.ncbi.nlm.nih.gov/pubmed/ 30419423

Publisher's Note Springer Nature remains neutral with regard to jurisdictional claims in published maps and institutional affiliations. 